\title{
Association between mRNA levels of DNMT1, DNMT3A, DNMT3B, MBD2 and LINE-1 methylation status in infants with tetralogy of Fallot
}

\author{
WEI SHENG ${ }^{1,2}$, YANYAN QIAN ${ }^{2}$, HUIJUN WANG $^{1}$, XIAOJING MA $^{1}$, PING ZHANG $^{1}$, \\ LONG CHEN $^{3}$, DUAN MA ${ }^{1,2,4}$ and GUOYING HUANG ${ }^{1,4}$ \\ ${ }^{1}$ Children's Hospital, Fudan University, Shanghai 201102; ${ }^{2}$ Key Laboratory of Molecular Medicine, \\ Ministry of Education, Shanghai Medical College, ${ }^{3}$ Department of Forensic Medicine, \\ ${ }^{4}$ Birth Defects Research Center, Fudan University, Shanghai 200032, P.R. China
}

Received March 18, 2013; Accepted June 14, 2013

DOI: $10.3892 / \mathrm{ijmm} .2013 .1427$

\begin{abstract}
DNA methylation is catalyzed and maintained by DNA methyltransferases (DNMTs: DNMT1, DNMT3A and DNMT3B) and methyl-CpG-binding domain protein 2 (MBD2). However, little is known about the biological and clinical significance of the expression changes of DNMTs and MBD2 and their association with the methylation levels of long interspersed nuclear element-1 (LINE-1) in patients with tetralogy of Fallot (TOF). In this study, quantitative RT-PCR (qRT-PCR) was applied to analyze the mRNA levels of DNMTs and MBD2. The methylation status of LINE-1 was measured using the sequenom MassARRAY platform. The mRNA levels of the DNMTs and MBD2 showed a statistically significant decrease in the patients with TOF $(\mathrm{P}<0.001)$. The results also showed that patients with TOF had significantly lower global DNA methylation levels with a median of $61.50 \%$ [interquartile range (IQR), 59.78-63.77] compared with 63.54\% (IQR, 62.49-64.88) among the controls $(\mathrm{P}=0.0099)$. In the controls, only DNMT1 showed a significant positive correlation with the DNMT3A mRNA levels $(\mathrm{r}=0.718, \mathrm{P}=0.002)$. Of note, the DNMT1, DNMT3A, DNMT3B and MBD2 mRNA levels positively correlated with each other; this was statistically significant $(\mathrm{P}<0.05)$. A significant positive correlation with the global DNA methylation status was observed only for MBD2 $(\mathrm{r}=-0.579, \mathrm{P}=0.005)$ in patients with TOF. In conclusion, lower LINE-1 methylation levels significantly correlate with aberrant MBD2 mRNA levels. The lower expression of DNMT1 and DNMT3B may play an important role in the pathogenesis of TOF.
\end{abstract}

Correspondence to: Professor Duan Ma or Professor Guoying Huang, Children's Hospital, Fudan University, 399 Wanyuan Road, Shanghai 201102, P.R. China

E-mail: duanma@yahoo.cn

E-mail: gyhuang@shmu.edu.cn

Key words: DNA methyltransferase, long interspersed nuclear element-1 methylation, methyl-CpG-binding domain protein 2, tetralogy of Fallot

\section{Introduction}

Congenital heart defects (CHDs) are the most common type of major birth defect, and account for the majority of morbidity and mortality related to birth defects (1). Tetralogy of Fallot (TOF) is the most commonly observed conotruncal congenital heart defect, accounting for $10 \%$ of all CHDs, with an incidence of 3.6 per 10,000 live births (2). The treatment of these patients has evolved significantly over the last few decades (3); however a genetic explanation, particularly epigenetic factors, is lacking for the failure of cardiac development in the majority of children with TOF. Epigenetic changes refer to the heritable changes in genome function that occur without a change in primary DNA sequence, characterized by covalent modifications of cytosine bases and histones, and changes in the positioning of nucleosomes (4). They are fundamental to the regulation of many cellular processes, including gene and microRNA expression, DNA-protein interactions, the suppression of transposable element mobility, cellular differentiation, embryogenesis, $\mathrm{X}$-chromosome inactivation and genomic imprinting (5).

Currently, the most widely studied epigenetic modification in humans is DNA methylation. DNA methylation occurs almost exclusively in the context of $\mathrm{CpG}$ dinucleotides, which control the transcriptional activity of genes by various mechanisms (6). The hypomethylation of genomic DNA has been reported to contribute to chromosome instability and to alter gene expression, cell differentiation and apoptosis during embryogenesis (7). Long interspersed nuclear element-1 (LINE-1) constitutes $17-25 \%$ of the human genome (8). Since LINE-1 sequences are highly repeated and widely interspersed human retrotransposons, their methylation level can serve as a surrogate marker for global genomic DNA methylation (9). LINE-1 hypomethylation is frequently observed in certain diseases, such as colon cancer (10), neural tube defects (NTDs) (11) and systemic lupus erythematosus (SLE) (12). LINE-1 hypomethylation have been proven to be associated with the increased occurrence of non-syndromic CHDs (9). We have previously shown that lower LINE-1 methylation levels are associated with an increased risk of TOF (13). However, the 
molecular mechanisms leading to LINE-1 hypomethylation in patients with TOF are not yet well understood.

DNA methylation is catalyzed and maintained by a family of DNA methyltransferases (DNMTs), including DNMT1, DNMT3A and DNMT3B. DNMT1 is known as a maintenance DNMT; it has a high preference for hemimethylated DNA substrates and maintains methylation patterns during DNA replication and accurately replicates genomic DNA methylation patterns during cell division in mammalian cells (14). DNMT3A and DNMT3B are responsible for the de novo methylation of DNA during early embryogenesis, thereby establishing a somatic methylation pattern (15). Methyl-CpG-binding domain protein 2 (MBD2) is one of the methyl-CpG binding proteins (MBPs); it binds methylated cytosine and attracts chromatin inactivation complexes containing histone deacetylase. Moreover, MBD2 has also been shown to possess demethylase activity (16). The aberrant expression of DNMTs and MBD2 may influence the DNA methylation pattern and consequently lead to diseases. The overexpression of DNMTs has been found in several types of cancer, such as lung cancer (17), breast cancer (18) and hepatocellular carcinoma (19). Moreover, lower mRNA levels of DNMTs have been reported in patients with spermatogenic arrest (20), systemic lupus erythematosus (21) and atopic dermatitis (22). Of note, transcript levels of DNMTs, showing no differences between patients and controls, have also been reported in patients with systemic lupus erythematosus (23). However, little is known about the variations in the mRNA levels of DNMT1, DNMT3A, DNMT3B and MBD2 and their association with the LINE-1 methylation status in patients with TOF.

In this study, to obtain a deeper understanding of the role of epigenetic mechanisms in patients with TOF, we measured and compared the mRNA levels of DNMTs and MBD2 and the methylation pattern of LINE-1 in normal and TOF samples. The association between the transcript levels of DNMTs, MBD2 and the LINE-1 methylation status was also investigated in normal and TOF samples.

\section{Materials and methods}

Patients and controls. In this study, 48 patients with TOF were recruited from the Children's Hospital of Fudan University, Shanghai, China, including 31 males (64.58\%) and 17 females (35.42\%), age ranging from 1 to 48 months $(14.88 \pm 11.27$, mean \pm SD). Patients were diagnosed by an echocardiogram, and the diagnoses were confirmed by surgery. Normal heart tissue samples were obtained from autopsy cases at the Department of Forensic Medicine, Fudan University. Samples from 16 healthy control subjects who had died by traffic accidents were also recruited in this study, including samples from 10 males $(62.50 \%)$ and 6 females $(37.50 \%)$, age ranging from 0.5 to 38 years $(20.91 \pm 14.22$, mean \pm SD). The clinical features of the study subjects are summarized in Table I. To exclude the tissue heterogeneity that may affect methylation levels, all the tissue samples obtained from right ventricular outflow tracts were saved in RNAlater ${ }^{\circledR}$ (Ambion, Inc., Austin, TX, USA) immediately after surgical resection or autopsy and stored until use.

This study was approved by the local Ethics Committee of Fudan University. Written informed consent was obtained from the parents and relatives of all the study participants.
Table I. Clinical characteristics of TOF and control samples.

\begin{tabular}{lcc}
\hline Characteristic & TOF $(\mathrm{n}=48)$ & Control $(\mathrm{n}=16)$ \\
\hline Age (mean \pm SD) & $\begin{array}{c}14.88 \pm 11.27 \\
(\text { months })\end{array}$ & $\begin{array}{c}20.91 \pm 14.22 \\
\text { (years) }\end{array}$ \\
Gender, n $(\%)$ & & \\
Male & $31(64.58)$ & $11(68.75)$ \\
Female & $17(35.42)$ & $5(31.25)$ \\
\hline
\end{tabular}

TOF, tetralogy of Fallot.

RNA extraction and quantitative RT-PCR ( $R R T-P C R)$. Total RNA was extracted from the heart tissue samples using TRIzol reagent (Invitrogen, Carlsbad, CA, USA) according to the manufacturer's instructions. RNA was reverse-transcribed using the PrimeScript RT reagent kit with gDNA Eraser (Perfect Real Time) (Takara Bio, Inc., Shiga, Japan) and the integrity of the synthesized cDNA was confirmed using glyceraldehyde 3-phosphate dehydrogenase (GAPDH) as an endogenous control. qRT-PCR was performed using SYBR Premix Ex Taq GC (Perfect Real Time) (Takara Bio, Inc.) in a $10 \mu \mathrm{l}$ reaction volume, containing $5 \mu \mathrm{l}$ SYBR Premix Ex Taq GC, $0.2 \mu \mathrm{M}$ of each primer, 0.2 unit ROX1 Reference Dye and $100 \mathrm{ng}$ cDNA. The reactions were performed in triplicate and analyzed using an ABI 7900 Sequence Detection System (Applied Biosystems, Carlsbad, CA, USA). Relative expression levels were calculated according to the standard $2^{-\Delta \Delta \mathrm{Ct}}$ method using beta-2 microglobulin (B2M) and the GAPDH gene as an endogenous control for normalization. The primer sequences used in qRT-PCR analysis are listed in Table II.

DNA extraction and sodium bisulfite conversion. Genomic DNA was extracted from the heart tissue samples using a QIAamp DNA Micro kit (Qiagen, Hilden, Germany) according to the manufacturer's instructions. The concentration and purity of the DNA were determined by absorbance at 260 and 280 nm using a NanoDrop ${ }^{\mathrm{TM}} 1000$ Spectrophotometer (Thermo Scientific, Wilmington, MA, USA) on an agrose gel. Sodium bisulfite modification for the extracted DNA was performed using an EZ DNA Methylation kit ${ }^{\mathrm{TM}}$ (Zymo Research Corp., Orange, CA, USA) according to the manufacturer's instructions. The bisulfite-converted DNA was resuspended in $10 \mu \mathrm{l}$ elution buffer and stored at $-80^{\circ} \mathrm{C}$ until the samples were ready for use.

MassARRAY quantitative methylation analysis. The Sequenom MassARRAY platform was used to perform the quantitative methylation analysis of LINE-1 as previously described (13). The primers used in this study were designed using EpiDesigner (http://epidesigner.com). Based on the analyzed information for the LINE-1 promoter region, we designed the primers as follows: forward, cagtaatacgactcactatagggagaagg-TTTTATT AGGGAGTGTTAGATAGTGGG and reverse, aggaagagagCCCCAAAAATAAAACCTACAAAAAC. The analyzed sequence represents a 468 base pair fragment (positions 835-386) in the 5'-untranslated region (5'-UTR) of the LINE-1 element. 
Table II. Primer sequences and product lengths for qPT-PCR analysis.

\begin{tabular}{lllc}
\hline Genes & \multicolumn{1}{c}{ Forward primer $\left(5^{\prime} \rightarrow 3^{\prime}\right)$} & \multicolumn{1}{c}{ Reverse primer $\left(5^{\prime} \rightarrow 3^{\prime}\right)$} & Product length $(\mathrm{bp})$ \\
\hline DNMT1 & AAACCCCTTTCCAAACCTCG & CTGGTGCTTTTCCTTGTAATCC & 101 \\
DNMT3A & CCAAGTTCAGCAAAGTGAGGAC & TGGACTGGGAAACCAAATACC & 145 \\
DNMT3B & TCCCAGCTCTTACCTTACCATC & ATCTCCACTGTCTGCCTCCA & 151 \\
MBD2 & TTCAAGGAGTTGGTCCAGGTAG & GCAGGGTTCTTTTCCACAGC & 121 \\
B2M & TGCTGTCTCCATGTTTGATGTATCT & TCTCTGCTCCCCACCTCTAAGT & 161 \\
GAPDH & AGAAGGCTGGGGCTCATTTG & AGGGGCCATCCACAGTCTTC & 220 \\
\hline
\end{tabular}

DNMT, DNA methyltransferase; MBD2, methyl-CpG-binding domain protein 2; B2M, beta-2 microglobulin; GAPDH, glyceraldehyde 3-phosphate dehydrogenase.

Table III. mRNA level of DNMT1, DNMT3A, DNMT3B and MBD in the control samples and patients with TOF.

\begin{tabular}{|c|c|c|c|c|}
\hline Genes & Control (mean $\pm \mathrm{SD}, \mathrm{n}=16)$ & $\mathrm{TOF}($ mean $\pm \mathrm{SD}, \mathrm{n}=48)$ & Difference & P-value ${ }^{a}$ \\
\hline DNMT1 & $4.186 \pm 2.77$ & $1.245 \pm 0.791$ & $2.941 \pm 1.78$ & $<0.0001$ \\
\hline DNMT3A & $2.797 \pm 1.78$ & $1.638 \pm 0.692$ & $1.159 \pm 1.24$ & 0.0241 \\
\hline DNMT3B & $17.31 \pm 21.21$ & $1.678 \pm 3.67$ & $15.63 \pm 12.45$ & $<0.0001$ \\
\hline MBD2 & $2.450 \pm 1.78$ & $0.992 \pm 0.375$ & $1.459 \pm 1.08$ & 0.0007 \\
\hline
\end{tabular}

${ }^{a}$ Mann-Whitney test was performed. DNMT, DNA methyltransferase; MBD, methyl-CpG-binding domain protein; TOF, tetralogy of Fallot.

Briefly, bisulfite-treated DNA was amplified with primers and the PCR products were spotted on a 384-pad SpectroChip (Sequenom, Inc., San Diego, CA, USA) and followed by spectral acquisition on a MassARRAY analyzer. The spectra and the methylation values of matrix-associated laser desorption/ionization time-of-flight mass spectrometry (Sequenom, Inc.) were collected and analyzed using EpiTYPER software (version 1.0; Sequenom, Inc.).

Statistical analysis. Data were analyzed using GraphPad Prism (version 5.0; GraphPad Software Inc., San Diego, CA, USA) and SPSS software (version 13.0; SPSS Inc., Chicago, IL, USA). The Mann-Whitney U test was performed to evaluate the significance of any differences between the TOF and control groups. Spearman's rank correlation was used to examine the correlation between two continuous variables. All statistical analyses were two-sided and a P-value $<0.05$ was considered to indicate a statistically significant difference.

\section{Results}

mRNA levels of DNMT1, DNMT3A, DNMT3B and MBD2 in patients with TOF and the control samples. We performed qRT-PCR to determine the expression levels of DNMT1, DNMT3A, DNMT3B and MBD2 genes in the 48 TOF and 16 normal samples. The patients with TOF had statistically significant lower mRNA levels of DNMTs (DNMT1, DNMT3A and DNMT3B) compared with the controls $(\mathrm{P}<0.001)$. The MBD2 gene also showed a significantly decreased mRNA level in the TOF samples $(\mathrm{P}<0.001)$ (Table III). Of note, the DNMT1 and DNMT3B mRNA levels were significantly decreased in the TOF samples compared with the normal controls $(\mathrm{P}<0.0001)$. Moreover, in the control group, when the age was taken into account, Spearman's rho correlation coefficients were obtained with respect to all the enzymes: $\mathrm{r}=0.046, \mathrm{P}=0.876$ (DNMT1); $\mathrm{r}=0.169, \mathrm{P}=0.530$ (DNMT3A); $\mathrm{r}=-0.221, \mathrm{P}=0.411$ (DNMT3); $\mathrm{r}=-0.013, \mathrm{P}=0.961$ (MBD2); however, no significant correlations were observed. In the patient groups, two significant negative correlations with age were observed for DNMT1 $(\mathrm{r}=-0.327, \mathrm{P}=0.023)$ and DNMT3A $(\mathrm{r}=-0.292, \mathrm{P}=0.044)$, while DNMT3B and MBD2 showed no significant correlation $(r=-0.069, P=0.640 ; r=-0.077, P=0.605$; respectively). When considering the gender difference, we found no significant difference or correlation between DNMT1, DNMT3A, DNMT3B and MBD2 in the control and TOF samples $(\mathrm{P}>0.05)$.

Analysis of the correlation between the $M R N A$ levels of $D N M T 1, D N M T 3 A, D N M T 3 B$ and MBD2. To determine the correlation between the mRNA levels of DNMT1, DNMT3A, DNMT3B and MBD2 in the control samples and patients with TOF, we performed several Spearman's correlation tests by SPASS 13.0. In the control samples, a statistically significant positive correlation was observed only between DNMT1 and DNMT3A ( $r=0.718, P=0.002$ ) (Fig. 1). However, DNMT1 had a significant positive correlation with DNMT3A ( $r=0.677$, $\mathrm{P}<0.0001)$, DNMT3B ( $\mathrm{r}=0.407, \mathrm{P}=0.004)$ and MBD2 ( $\mathrm{r}=0.636$, $\mathrm{P}<0.0001)$ in the group of TOF patients. Moreover, DNMT3A also showed a significant positive correlation with DNMT3B $(\mathrm{r}=0.258, \mathrm{P}=0.050)$ and MBD2 ( $\mathrm{r}=0.493, \mathrm{P}<0.0001)$; $\mathrm{DNMT3B}$ had a significant positive correlation with MBD2 $(r=0.641$, $\mathrm{P}<0.0001$ ) in the patients with TOF (Fig. 2). 

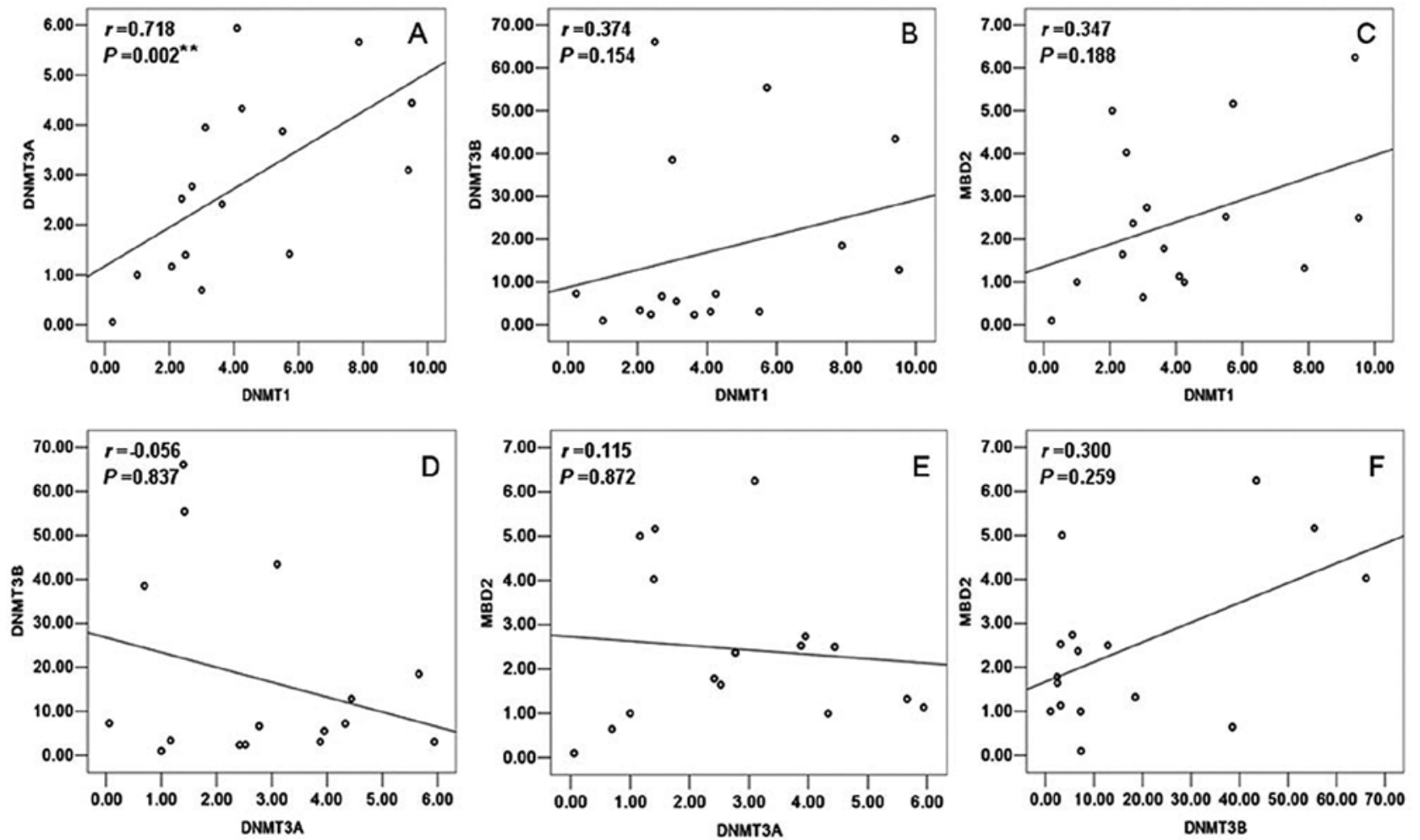

Figure 1. Correlation between the mRNA levels of DNA methyltransferase (DNMT)1, DNMT3A, DNMT3B and MBD2 in the normal control samples. (A) DNMT1 and DNMT3A, (B) DNMT1 and DNMT3B, (C) DNMT1 and methyl-CpG-binding domain protein 2 (MBD2), (D) DNMT3A and DNMT3B,

(E) DNMT3A and MBD2, (F) DNMT3B and MBD2. Statistically significant values are marked with an asterisk.
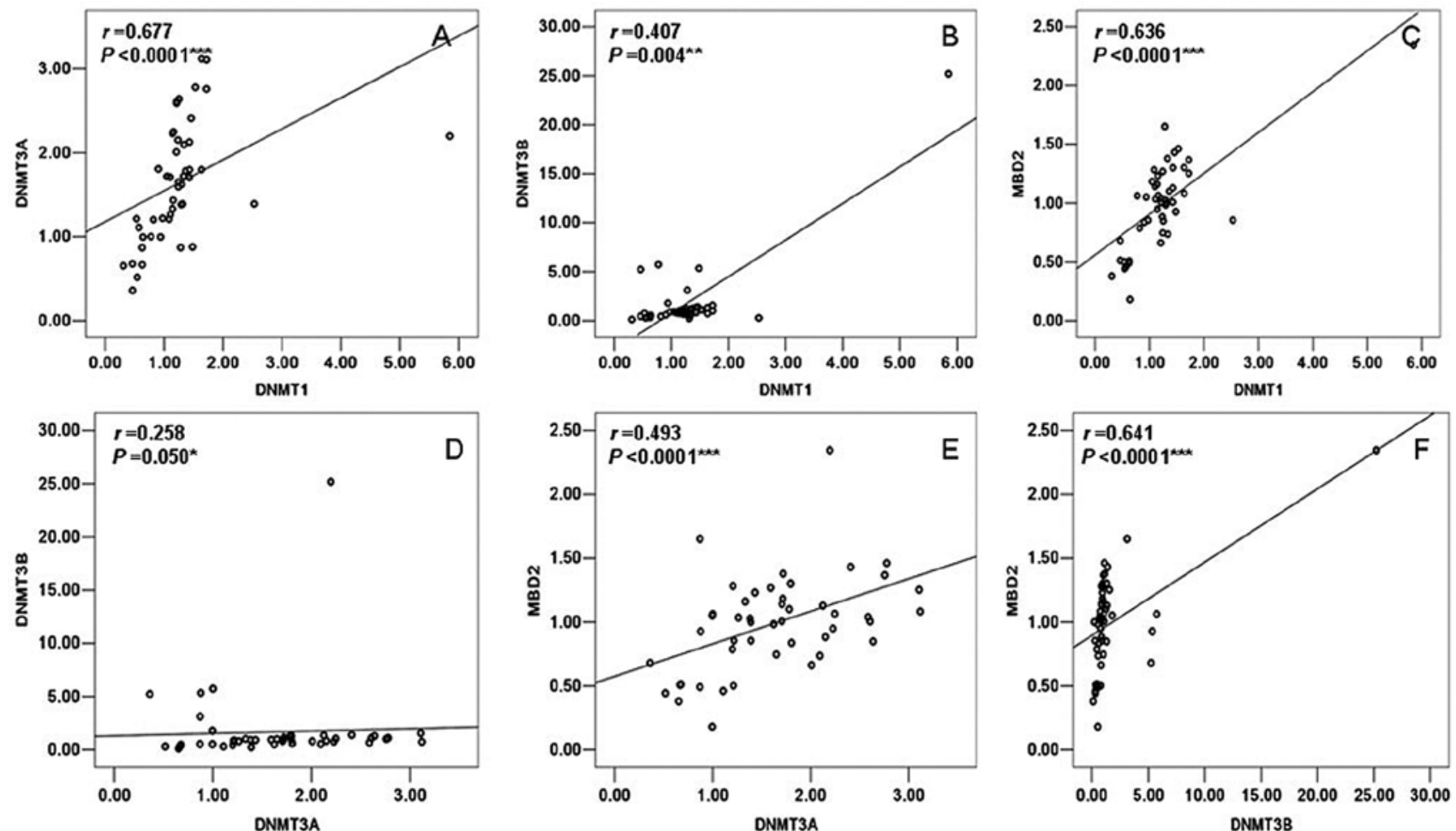

Figure 2. Correlation between the mRNA levels of DNA methyltransferase (DNMT)1, DNMT3A, DNMT3B and methyl-CpG-binding domain protein 2 (MBD2) in patients with tetralogy of Fallot. (A) DNMT1 and DNMT3A, (B) DNMT1 and DNMT3B, (C) DNMT1 and MBD2, (D) DNMT3A and DNMT3B, (E) DNMT3A and MBD2, (F) DNMT3B and MBD2. Statistically significant values are marked with an asterisk.

LINE-1 methylation levels in patients with TOF and the control samples. To explore the global DNA methylation status, we analyzed the methylation status of LINE-1 in a similar region of the cardiac samples obtained from 22 patients 


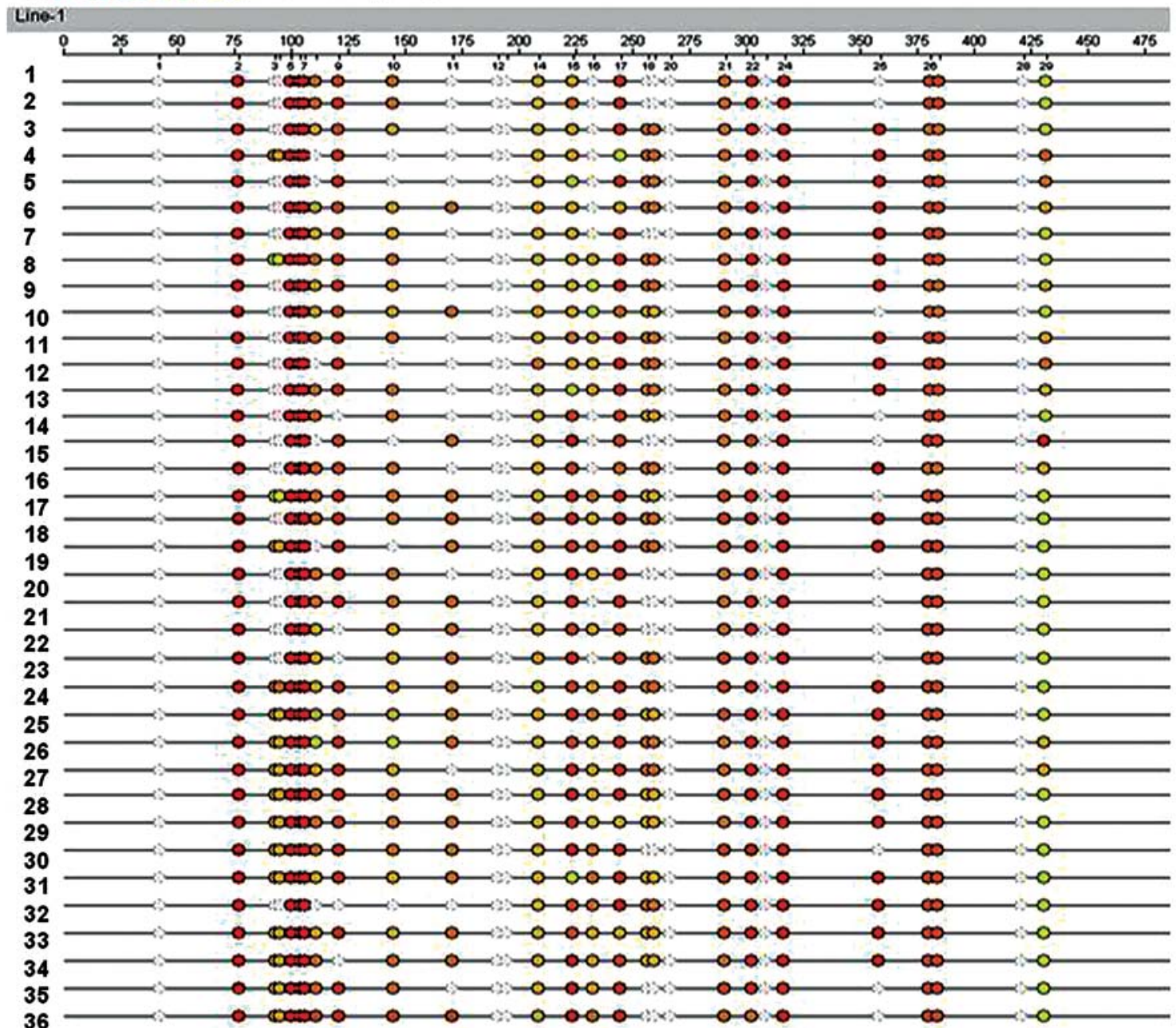

B

LINE-1

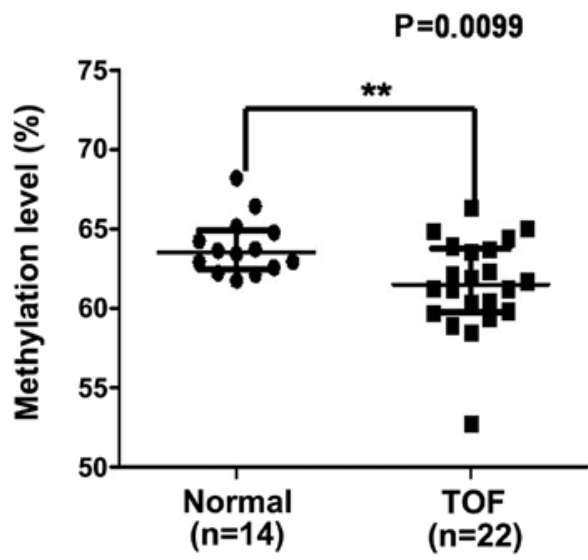

Figure 3. Sequenom MassARRAY analysis of the percentage of interspersed nuclear element-1 (LINE-1) DNA methylation. (A) The epigram shows the percentage of the DNA methylation levels of each CpG site of the target region. Different colors display relative methylation changes in $10 \%$ increments. The yellow circle indicates 100\% methylation, and the red circle is 0\% methylation at each CpG site. Cases 1-14 represent normal heart tissue, cases 15-36 represent tetralogy of Fallot (TOF) patients. (B) Median methylation levels of LINE-1 between the normal samples and patients with TOF. Control, $\mathrm{n}=14$; TOF, $\mathrm{n}=22$. Mann-Whitney test was performed, ${ }^{* *} \mathrm{P}<0.01$.

with TOF and 14 control samples. The methylation level of LINE-1 was significantly lower in the patients with TOF with a median of $61.50 \%$ [interquartile range (IQR), 59.78-63.77] compared with $63.54 \%$ (IQR, 62.49-64.88) among the controls $(\mathrm{P}=0.0099)$ (Fig. 3).
Moreover, taking into consideration the age or gender differences in the individual samples, we analyzed the correlation between age or gender and the LINE-1 methylation status in the normal and TOF samples. In the normal group, no correlation between age and the LINE-1 methylation level 

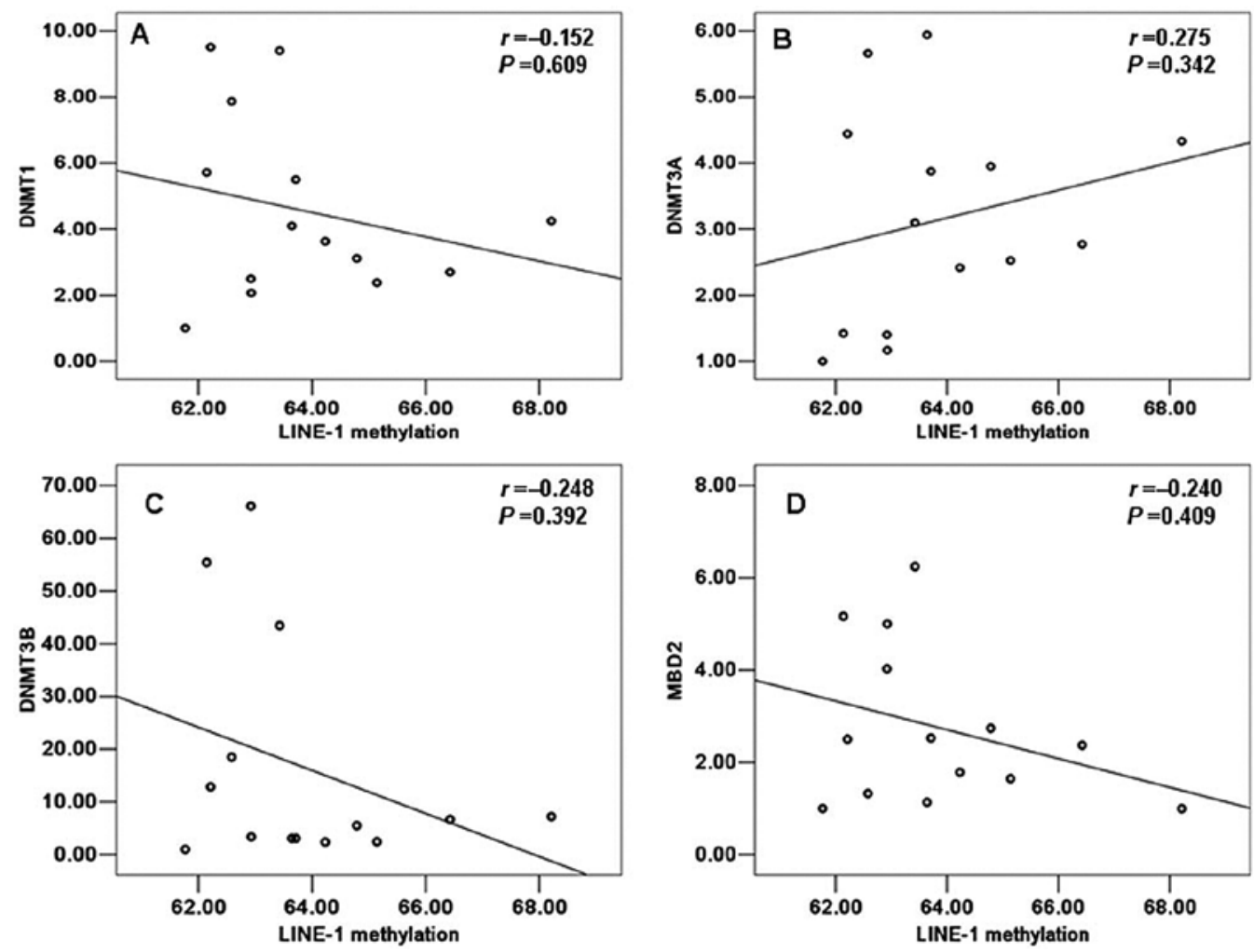

Figure 4. Correlation between interspersed nuclear element-1 (LINE-1) methylation indices and mRNA levels of (A) DNA methyltransferase (DNMT)1, (B) DNMT3A, (C) DNMT3B and (D) methyl-CpG-binding domain protein 2 (MBD2) in normal control samples. Statistically significant values are marked with an asterisk.

was observed $(\mathrm{r}=0.2709, \mathrm{P}=0.3488)$. The males and females had similar methylation values (63.32 vs. $63.54 \%$, median) and showed no significant difference $(\mathrm{P}=0.9527)$. In the TOF patient panels, there was no correlation observed between age and the methylation status of LINE-1 ( $\mathrm{r}=-0.3980, \mathrm{P}=0.0666)$. Although the females showed a higher methylation level than the males (62.04 vs. $61.25 \%$, median), no statistically significant difference was observed $(\mathrm{P}=0.7074)$.

Correlation between the mRNA levels of DNMT1, DNMT3A, DNMT3B and MBD2 and LINE-1 methylation status. To ascertain whether a correlation exists between the LINE-1 methylation status and the mRNA levels of DNMT1, DNMT3A, DNMT3B and MBD2 and whether such a correlation exists in both the control and TOF samples, we analyzed the data from the same individual from 14 normal and $22 \mathrm{TOF}$ samples by SPASS 13.0. A positive correlation with the LINE-1 methylation status was detected only for DNMT3A in the control group, but no statistically significant value was observed ( $\mathrm{r}=0.275, \mathrm{P}=0.342$ ) (Fig. 4). Moreover, in the control group, DNMT1, DNMT3B and MBD2 all a showed negative correlation with the LINE-1 methylation status and this was not statistically significant $(\mathrm{r}=-0.152, \mathrm{P}=0.609 ; \mathrm{r}=-0.248, \mathrm{P}=0.392$; $\mathrm{r}=-0.240, \mathrm{P}=0.409$; respectively). However, on the contrary, in the group of TOF patients, DNMT3A showed a negative correlation with the LINE-1 methylation status but this was not statistically significant $(\mathrm{r}=-0.124, \mathrm{P}=0.584)$ (Fig. $5 \mathrm{~B})$. Of note, we also observed a negative correlation between the LINE-1 methylation status and the mRNA levels of DNMT1,
DNMT3B and MBD2 in the patients with TOF which was similar to that in the normal samples. However, only MBD2 showed a statistically significant correlation with the LINE-1 methylation status $(\mathrm{r}=-0.579, \mathrm{P}=0.005)$ (Fig. 5D). Moreover, DNMT1, DNMT3B did not show a significant correlation with the LINE-1 methylation status $(\mathrm{r}=-0.252, \mathrm{P}=0.257$; $\mathrm{r}=-0.243$, $\mathrm{P}=0.275$; respectively) (Fig. 5A and $\mathrm{C}$ ).

\section{Discussion}

In this study, we demonstrated that patients with TOF had significantly lower global DNA methylation levels than those of the controls, which may be associated with the complex etiology of TOF. Moreover, the mRNA levels of the DNMTs (DNMT1, DNMT3A and DNMT3B) and the MBD2 gene were significantly decreased in the TOF samples; in particular, the decrease in the levels of DNMT1 and DNMT3B was more significant. A significant negative correlation between the LINE-1 methylation status and the MBD2 mRNA levels was observed in the patients with TOF. These findings may provide important insight into the development of pathologies from an epigenetic viewpoint.

DNA methylation, the most investigated epigenetic hallmark, is a reversible mechanism which modifies genome function and chromosomal stability through the addition of methyl groups to cytosine located in $\mathrm{CpG}$ dinucleotides to form 5 methylcytosine ( $5 \mathrm{mC})$. The $\mathrm{CpG}$ dinucleotides tend to cluster in regions termed CpG islands (24). Unlike DNA sequence mutations, the inheritance patterns of these epigenetic events in humans are poorly 

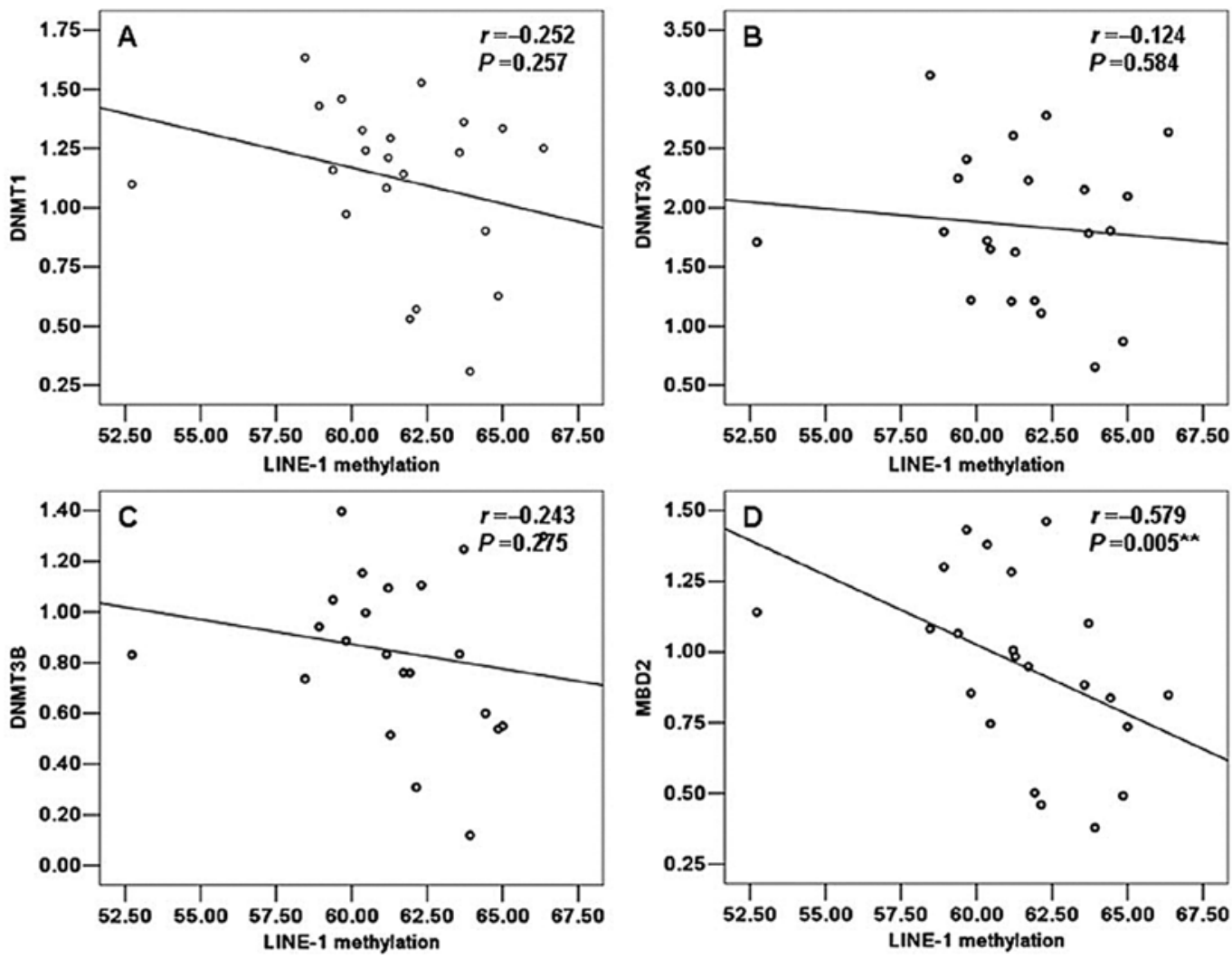

Figure 5. Correlation between interspersed nuclear element-1 (LINE-1) methylation indices and mRNA levels of (A) DNA methyltransferase (DNMT)1, (B) DNMT3A, (C) DNMT3B and (D) MBD2 in patients with tetralogy of Fallot. Statistically significant values are marked with an asterisk.

understood. The hypomethylation of the global genome largely promotes chromosomal instability, translocations, gene disruption and reactivation of endoparasitic sequences (25). LINE-1 methylation patterns may serve as a potential indicator of global DNA methylation (11), which has been proven to be frequently hypomethylated in several types of cancer (26). Moreover, maternal LINE-1 hypomethylation has also been found to be associated with an increased occurrence of non-syndromic CHDs (9). Of note, higher methylation levels of LINE-1 have also been reported in patients with Alzheimer's disease (AD) (27). These findings suggest that changes in the LINE-1 methylation status may not be restricted to cancers but may be present in other diseases and may show hypo- or hype-methylation status under different conditions. In the present study, we found that patients with TOF had significantly lower levels of LINE-1 methylation compared with samples from normal subjects, suggesting that reduced LINE-1 methylation levels may increase the chromosomal instability and consequently alter the expression of genes associated with heart development, eventually leading to TOF. However, the exact molecular mechanisms of LINE-1 hypomethylation associated with the etiology of TOF remain unclear and require further study.

DNA methylation is regulated mainly by four DNMTs (DNMT1, DNMT2, DNMT3A and DNMT3B). MBD2 possesses demethylase activity and participates in DNA methylation and demethylation events (16). In the present study, we found that the mRNA levels of DNMT1, DNMT3A, DNMT3B and MBD2 were significantly decreased in patients with TOF; in particular, the decrease in the levels of DNMT1 and DNMT3B was more significant compared with the normal control samples. It is known that lower mRNA levels of DNMT1 and DNMT3B eliminate methyltransferase activity and thereby reduce DNA methylation by $>95 \%$ (28). DNA methylation requires cooperative interactions of DNMT1 and DNMT3B. Thus, we hypothesized that global DNA hypomethylation in patients with TOF may be induced by the simultaneous decrease in the mRNA levels of DNMT1 and DNMT3B. Moreover, due to the demethylase activity of MBD2, the increased mRNA level of MBD2 is considered to contribute to global DNA hypomethylation (29). By contrast, we observed the decreased expression of MBD2 mRNA in patients with TOF; thus, we hypothesized that the decreased expression of MBD2 mRNA may be due to the global DNA hypomethylation (due to feedback mechanisms) in patients with TOF.

In this study, we only observed a significant positive correlation between the mRNA levels of DNMT1 and DNMT3A and no correlation was observed among the other DNMTs and MBD2 in the normal samples; this suggests that changes in the expression of DNMT1 and DNMT3A may occur simultaneously. On the contrary, in the patients with TOF, we found that the mRNA levels of DNMT1,DNMT3A,DNMT3B and MBD2 positively correlated with each other and this was statistically significant. Moreover, no correlation was observed between age and the mRNA levels of the DNMTs and MBD2 in the normal group; however, a significant negative correlation with age was observed for DNMT1 and DNMT3A in the patient group; this result is in accordance with the study by Zhang et al (30). No significant correlation with gender was observed among 
DNMT1, DNMT3A, DNMT3B and MBD2 in the control and TOF samples. Furthermore, we found no correlation between LINE-1 hypomethylation and the mRNA levels of the DNMTs and MBD2 in the normal group; however, a significant negative correlation between the LINE-1 hypomethylation and the MBD2 mRNA level was observed in the patient group. It has been reported that the decrease in DNMT activity is due to the decrease in the mRNA levels of DNMTs (31). However, a previous study suggested that the DNMT1 protein levels were elevated in human breast cancer cells due to increased stability, while the DNMT1 mRNA levels were unaltered (32). Luo et al (33) found that the mRNA levels of other enzymes (MBD1, MBD2, MBD3 and MBD4) involved in the DNA methylation process were significantly higher in patients with lupus erythematosus. Moreover, although patients with SLE have been shown to have significantly lower levels of DNMT1 mRNA than the controls, a correlation between the mRNA levels of DNMT1 and global DNA methylation was not observed (21). A recent study also suggested that there was no significant correlation between the global methylation levels and DNMT1 and MBD2 mRNA expression in patients with active and inactive SLE (29). However, in our study, we found that the reduced mRNA level of MBD2 had a significant correlation with global DNA hypomethylation in the patient group. We concluded that MBD2 may influence the DNA methylation pattern through an indirect method; the exact mechanisms involved require further investigation in the future.

As heat tissue samples are difficult to collect from healthy controls and TOF patients, one limitation of this study was that we were unable to obtain enough complete age-matched samples. Thus, the findings presented in our study require further investigation and validation in larger sample sizes. Moreover, based only on these data from clinical samples, we cannot ascertain whether methylation changes that are noted occur after the heart is already formed or after heart defect already exists. At the same time, we cannot determine whether these changes are reflective of the disease physiology or related to disease etiology. All the related research will be further explored using cell lines or animal models in future studies.

In conclusion, global DNA hypomethylation is one of the possible epigenetic variations associated with the complex etiology of TOF and significantly correlates with the aberrant expression of MBD2 mRNA in patients with TOF. The decreased expression of DNMT1 and DNMT3B mRNA may play an important role in the pathogenesis of TOF. The mechanisms of global DNA hypomethylation in patients with TOF are complex. Enzymes that participate in DNA methylation and demethylation events should be investigated further in a larger number of samples, and may thus provide important insight into the development of novel treatments for TOF, as well as provide a deeper understanding of the etiology of congenital heart disease.

\section{Acknowledgements}

This study was supported by grants from the National Basic Research Program of China (973 Program; 2010CB529504 and 2009CB941704) and the Key Program of the National Natural Science Foundation of China (30930096).

\section{References}

1. Bittel DC, Butler MG, Kibiryeva N, et al: Gene expression in cardiac tissues from infants with idiopathic conotruncal defects. BMC Med Genomics 4: 1, 2011.

2. Bedard E, McCarthy KP, Dimopoulos K, Giannakoulas G, Gatzoulis MA and Ho SY: Structural abnormalities of the pulmonary trunk in tetralogy of fallot and potential clinical implications: a morphological study. J Am Coll Cardiol 54: 1883-1890, 2009.

3. Di Felice V and Zummo G: Tetralogy of fallot as a model to study cardiac progenitor cell migration and differentiation during heart development. Trends Cardiovasc Med 19: 130-135, 2009.

4. Rodenhiser D and Mann M: Epigenetics and human disease: translating basic biology into clinical applications. CMAJ 174: 341-348, 2006.

5. Portela A and Esteller M: Epigenetic modifications and human disease. Nat Biotechnol 28: 1057-1068, 2010.

6. Goll MG and Bestor TH: Eukaryotic cytosine methyltransferases. Annu Rev Biochem 74: 481-514, 2005.

7. Lees-Murdock DJ, De Felici M and Walsh CP: Methylation dynamics of repetitive DNA elements in the mouse germ cell lineage. Genomics 82: 230-237, 2003.

8. Weisenberger DJ, Campan M, Long TI, et al: Analysis of repetitive element DNA methylation by MethyLight. Nucleic Acids Res 33: 6823-6836, 2005.

9. Chowdhury S, Cleves MA, MacLeod SL, James SJ, Zhao W and Hobbs CA: Maternal DNA hypomethylation and congenital heart defects. Birth Defects Res A Clin Mol Teratol 91: 69-76, 2011.

10. Sunami E, de Maat M, Vu A, Turner RR and Hoon DS: LINE-1 hypomethylation during primary colon cancer progression. PLoS One 6: e18884, 2011.

11. Wang L, Wang F, Guan J, et al: Relation between hypomethylation of long interspersed nucleotide elements and risk of neural tube defects. Am J Clin Nutr 91: 1359-1367, 2010.

12. Nakkuntod J, Avihingsanon Y, Mutirangura A and Hirankarn N: Hypomethylation of LINE-1 but not Alu in lymphocyte subsets of systemic lupus erythematosus patients. Clin Chim Acta 412: 1457-1461, 2011.

13. Sheng W, Wang JH, Ma JX, et al: LINE-1 methylation status and its association with tetralogy of fallot in infants. BMC Med Genomics 5: 20, 2012.

14. Rajendran G, Shanmuganandam K, Bendre A, Muzumdar D, Goel A and Shiras A: Epigenetic regulation of DNA methyltransferases: DNMT1 and DNMT3B in gliomas. J Neurooncol 104: 483-494, 2011

15. Okano M, Bell DW, Haber DA and Li E: DNA methyltransferases Dnmt3a and Dnmt3b are essential for de novo methylation and mammalian development. Cell 99: 247-257, 1999.

16. Detich N, Theberge J and Szyf M: Promoter-specific activation and demethylation by MBD2/demethylase. J Biol Chem 277: 35791-35794, 2002.

17. Lin RK, Hsu HS, Chang JW, Chen CY, Chen JT and Wang YC: Alteration of DNA methyltransferases contributes to $5^{\prime} \mathrm{CpG}$ methylation and poor prognosis in lung cancer. Lung Cancer 55: 205-213, 2007.

18. Girault I, Tozlu S, Lidereau R and Bieche I: Expression analysis of DNA methyltransferases $1,3 \mathrm{~A}$, and 3B in sporadic breast carcinomas. Clin Cancer Res 9: 4415-4422, 2003.

19. Fan H, Zhao ZJ, Cheng J, Su XW, Wu QX and Shan YF: Overexpression of DNA methyltransferase 1 and its biological significance in primary hepatocellular carcinoma. World $\mathrm{J}$ Gastroenterol 15: 2020-2026, 2009.

20. Adiga SK, Ehmcke J, Schlatt S, et al: Reduced expression of DNMT3B in the germ cells of patients with bilateral spermatogenic arrest does not lead to changes in the global methylation status. Mol Hum Reprod 17: 545-549, 2011.

21. Zhu XH, Liang J, Li F, Yang YS, Xiang LH and Xu JH: Analysis of associations between the patterns of global DNA hypomethylation and expression of DNA methyltransferase in patients with systemic lupus erythematosus. Int J Dermatol 50: 697-704, 2011.

22. Nakamura T, Sekigawa I, Ogasawara H, et al: Expression of DNMT-1 in patients with atopic dermatitis. Arch Dermatol Res 298: 253-256, 2006.

23. Balada E, Ordi-Ros J, Serrano-Acedo S, Martinez-Lostao L, Rosa-Leyva M and Vilardell-Tarrés M: Transcript levels of DNA methyltransferases DNMT1, DNMT3A and DNMT3B in $\mathrm{CD}^{+} \mathrm{T}$ cells from patients with systemic lupus erythematosus. Immunology 124: 339-347, 2008. 
24. Crews D and McLachlan JA: Epigenetics, evolution, endocrine disruption, health, and disease. Endocrinology 147 (Suppl 6): S4-S10, 2006.

25. Gaudet F, Hodgson JG, Eden A, et al: Induction of tumors in mice by genomic hypomethylation. Science 300: 489-492, 2003

26. Wilson AS, Power BE and Molloy PL: DNA hypomethylation and human diseases. Biochim Biophys Acta 1775: 138-162, 2007.

27. Bollati V, Galimberti D, Pergoli L, et al: DNA methylation in repetitive elements and Alzheimer disease. Brain Behav Immun 25: 1078-1083, 2011.

28. Rhee I, Bachman KE, Park BH, et al: DNMT1 and DNMT3b cooperate to silence genes in human cancer cells. Nature 416: $552-556,2002$.

29. Liu CC, Ou TT, Wu CC, et al: Global DNA methylation, DNMT1, and MBD2 in patients with systemic lupus erythematosus. Lupus 20: 131-136, 2011.
30. Zhang Z, Deng C, Lu Q and Richardson B: Age-dependent DNA methylation changes in the ITGAL (CD11a) promoter. Mech Ageing Dev 123: 1257-1268, 2002.

31. Christman JK, Sheikhnejad G, Dizik M, Abileah S and Wainfan E: Reversibility of changes in nucleic acid methylation and gene expression induced in rat liver by severe dietary methyl deficiency. Carcinogenesis 14: 551-557, 1993.

32. Agoston AT, Argani P, Yegnasubramanian S, et al: Increased protein stability causes DNA methyltransferase 1 dysregulation in breast cancer. J Biol Chem 280: 18302-18310, 2005.

33. Luo Y, Li Y, Su Y, et al: Abnormal DNA methylation in T cells from patients with subacute cutaneous lupus erythematosus. Br J Dermatol 159: 827-833, 2008. 